\title{
TORSION DIFFERENTIALS AND DEFORMATION
}

\author{
BY
}

\author{
D. S. RIM (1)
}

ABSTRACT. Let $S$-scheme $X$ be a Schlessinger deformation of a curve $X_{0}$ defined over a field $k$. In $\S \S 1$ and 2 , the dimension of the parameter space $S$, the relative differentials of $X$ over $S$, and the fibres with singularity were studied, in case when $X_{0}$ is locally complete-intersection. In $\S 3$ we show that if $k$-scheme $X_{0}$ is a specialization of a smooth $k$-scheme, then the punctured spectrum Spex $\left(O_{X_{0}, x}\right)$ has to be connected for every point $x \in X_{0}$ such that $\operatorname{dim} O_{X_{0, x}} \geq 2$. In turn we construct a rigid singularity on a surface. In the last section a few conjectures amplifying those of $P$. Deligne are made.

Let $X_{0}$ be an algebraic variety over a fixed perfect field $k$. A (formal) deformation (2) of $X_{0}$ is meant by a pair $(R, X)$ where $R$ is a complete noetherian local $k$-algebra with the residue field $k$, and $X$ a flat (formal) $R$-scheme together with an isomorphism $X_{0} \leadsto k \otimes_{R} X$. A (formal) deformation $(R, X)$ of $X_{0}$ is called a (formal) versal deformation ( 3 ) of $X_{0}$ if every (formal) deformation of $X_{0}$ is induced from $(R, X)$. A (formal) versal deformation $(R, X)$ of $X_{0}$ such that $\operatorname{dim} \operatorname{Der}_{k}(R, k)$ is minimal will be called a minimally-versal (formal) deformation or a (formal) Schlessinger deformation of $X_{0}$. If $X_{0}$ is proper over $k$ or affine with isolated nonsmooth points only, we have the formal existence theorem due to $M$. Schlessinger, i.e. there exists a minimally-versal formal deformation of $X_{0}$. If $X_{0}$ is a projective variety with $H^{2}\left(X_{0}, \underline{O}_{X_{0}}\right)=0$ (for instance $X_{0}$ is a complete curve), then it follows from a theorem of $A$. Grothendieck that every formal deformation is "algebraisable" and in turn there exists a minimallyversal deformation of $X_{0}$.

Besides these existence theorems we have very little knowledge about the deformation of singularities at the present stage. In this paper we study the deformations of curves which is a relative complete-intersection over $k$, as well as rigid singularities on a surface. A complete-intersection, having no local obstruction for deformations, is the simplest case. Our method is based on a study of

Received by the editors April 10, 1971.

AMS 1970 subject classifications. Primary 14D15; Secondary 13 D10.

(1) The author acknowledges the financial support of I. H. E. S. as well as N. S. F.

(2) In general one considers deformations in which the characteristic also changes. Here we restrict ourselves to equicharacteristic case.

(3) One notes here the change of terminology from [8]. A minimally-versal deformation or a Schlessinger deformation in this paper was called a versal deformation in [8]. A change of the terminology comes from a necessity of considering a versal deformation in the sense of this paper which is not necessarily a minimal one. 
torsions and cotorsions of some modules carried out in $\$ 1$, which is of interest in itself but may be of no use in the case of noncomplete intersections. Nevertheless, our Theorem 2.7 may give an insight towards likely phenomenon in the general case. In a less precise language, we establish the following: Let $X_{0}$ be a reduced complete curve which is a relative complete-intersection over $k$, and let $(R, X)$ be a Schlessinger deformation of $X_{0}$. Then

(i) $\operatorname{dim} R=3 g-3+\operatorname{dim} H^{0}\left(X_{0}, \underline{\Omega}_{X_{0}}^{*}\right)$ where $g=\operatorname{dim} H^{1}\left(X_{0}, \underline{O}_{X_{0}}\right)$,

(i) ${ }^{\prime} \operatorname{dim} R-\operatorname{dim} H^{1}\left(X_{0}, \underline{\Omega}_{X_{0}}^{*}\right)=$ the dimension of the torsion $k$-differentials of $X_{0}$,

(ii) $\Omega_{X \mid R}$ has no torsion and $\Omega_{X \mid R}^{*}$ is an invertible sheaf on $X$,

(iii) $\operatorname{codim}\left(\operatorname{Sing}_{R}(X \mid R)\right.$ in $\left.\operatorname{Spec}(R)\right)=1$ where $\operatorname{Sing}_{R}(X \mid R)=\{z \in \operatorname{Spec}(R) \mid$ $X \rightarrow \operatorname{Spec}(R)$ is not smooth at the point $z$.

The properties (i) and (iii) are expected to be valid for an arbitrary reduced complete curve. For instance, a complete curve having ordinary multiple points with mutually transversal tangents does enjoy (i) and (iii), and considerably more, even though the parameter scheme $\operatorname{Spec}(R)$ is far from being regular in general [9].

In $\$ 3$ we are interested in the rigid isolated singularities. It has been known that there exists an affine variety (with an isolated singularity) which cannot be obtained as a specialization of a nonsingular variety. Indeed, H. Grauert and $\mathrm{H}$. Kerner have constructed a rigid isolated singularity of dimension $n$, provided $n \geq 4$ [4]. On the other hand, every complete reduced curve is conjecturally a specialization of a nonsingular curve, and therefore there cannot exist, conjecturally, a rigid 1-dimensional isolated singularity. Thus it raises the question if there exists a surface with a rigid isolated singularity. We first establish that every reduced surface with isolated singularities only which is obtained as a specialization of a nonsingular surface has to be unibranch at every point. Motivated by this fact, we construct a rigid isolated singularity on an irreducible rational surface. In the last section we have made a few conjectures based on our Theorem 2.7 as well as a number of empirical results. For instance, it will be shown in the forthcoming paper [9] that these conjectures are valid for any curve with ordinary multiple points (with mutually transversal tangents).

The author wishes to acknowledge his thanks to A. Grothendieck, P. Deligne as well as $\mathrm{H}$. Hironaka for the beneficial discussions.

1. Torsions and cotorsions. Let $A$ be a commutative noetherian ring. For any generically-free $A$-module $M$ of finite type, i.e. $K \otimes_{4} M$ is $K$-free, where $K$ is the total ring of fractions of $A$, we set $\mathrm{rg} M=$ the free rank of $K \otimes_{A} M$ as $K$-module. Given a generically-free $A$-module $M$ of finite type with $\operatorname{rg} M=d$, choose a finite presentation 


$$
0 \rightarrow N \rightarrow F \rightarrow M \rightarrow 0
$$

where $F$ is $A$-free of rank $m$. Since $N$ is a generically-free $A$-module of rank $m-d$, the natural map $\bigwedge^{m-d} N \otimes \bigwedge^{d} F \rightarrow \bigwedge^{m} F$ induces the map $\bigwedge^{m-u} N \otimes \bigwedge^{d} M \rightarrow \bigwedge^{m} F$ and hence the map $\alpha_{F}: \bigwedge^{d} M \rightarrow \operatorname{Hom}_{A}\left(\bigwedge^{m-d} N, \bigwedge^{m} F\right)$.

Lemma 1.0. For any generically-free A-module $M$ of finite type, the map $\alpha_{F}: \bigwedge^{d} M \rightarrow \operatorname{Hom}_{A}\left(\bigwedge^{m-d} N, \bigwedge^{m} F\right)$ does not depend on the choice of presentations of $M$.

Proof. Let

$$
\begin{aligned}
& 0 \rightarrow N_{1} \rightarrow F_{1} \rightarrow M \rightarrow 0, \\
& 0 \rightarrow N_{2} \rightarrow F_{2} \rightarrow M \rightarrow 0
\end{aligned}
$$

be any two finite presentations of $M$ where $\mathrm{rg} F_{i}=m_{i}$. Suppose that there exists an isomorphism

$$
\tau: \operatorname{Hom}_{A}\left({\stackrel{m}{1} \bigwedge^{-d} N_{1},}^{\Lambda^{\prime}} F_{1}\right) \rightarrow \operatorname{Hom}_{A}\left({ }^{m_{2}} \bigwedge^{-d} N_{2}, \stackrel{m}{\Lambda}^{2} F_{2}\right)
$$

with the following commutative diagram:

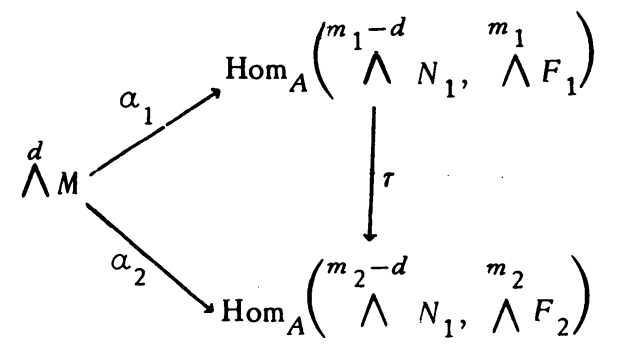

Then such $\tau$ must necessarily be unique since $\alpha_{i}(i=1,2)$ are generically isomorphisms, and $\operatorname{Hom}_{A}\left(, \bigwedge^{m} F\right)$ are torsion-free modules. Therefore it suffices to show an existence of such isomorphism $\tau$. Now, from Schannel's lemma, there exists an automorphism $\sigma \in$ Aut $_{A}\left(F_{1} \oplus F_{2}\right)$ with the commutative diagram:

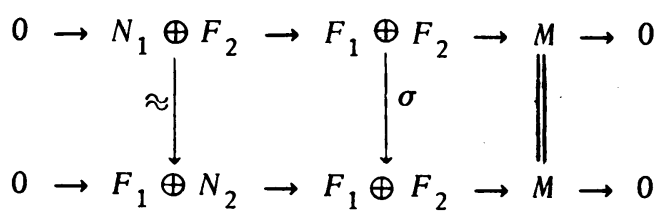

In turn, $\sigma$ induces a commutative diagram

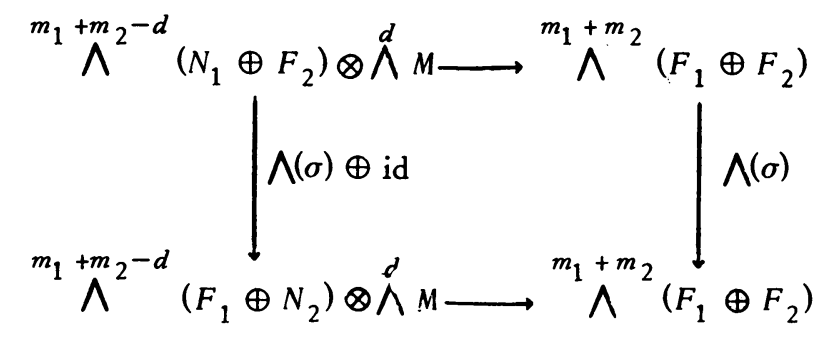


from which an existence of $\tau$ with a commutative diagram $(*)$ follows.

In view of the intrinsic nature of the map $\alpha: \bigwedge^{d} M \rightarrow \operatorname{Hom}_{A}\left(\bigwedge^{m-d} N, \bigwedge^{m} F\right)$, we shall denote this as $\alpha_{M}: \bigwedge^{d} M \rightarrow M_{\#}$ by setting $M_{\#}=\operatorname{Hom}_{A}\left(\bigwedge^{m-d} N, \Lambda^{m} F\right)$. We note the following simple properties of $M_{\#}$ which are trivial to verify.

(1) If we set $M_{\#}^{t}=\operatorname{Coker}\left(\bigwedge^{d} M \rightarrow M_{\#}\right)$, then

$$
0 \rightarrow(\stackrel{d}{\wedge} M)_{t} \rightarrow \stackrel{d}{\wedge} M \rightarrow M_{\#} \rightarrow M_{\#}^{t} \rightarrow 0
$$

is exact, where $\left(\bigwedge^{d} M\right)_{t}$ stands for the torsion submodule of $\bigwedge^{d} M$.

(2) $\alpha_{M}: \bigwedge^{d} M \rightarrow M_{\#}$ commutes with base change $A \rightarrow A^{\prime}$ provided $\operatorname{Tor}_{1}^{A}\left(M, A^{\prime}\right)=0$. In particular, (a) $S^{-1} A_{M} \rightarrow \alpha_{S^{-1} M}$ is an isomorphism for any multiplicative subset $S$ of $A$. (b) If $A$ is an $R$-algebra and $M$ is $R$-flat, then $\alpha_{R^{\prime}} \otimes_{R} M \rightarrow R^{\prime} \otimes_{R} \alpha_{M}$ is an isomorphism for any $R$-algebra $R^{\prime}$.

(3) If $M$ is $A$-free, then $\alpha_{M}$ is an isomorphism. Therefore Supp $M_{\#}^{t} \subset$ $\operatorname{Sing}_{A}(M)=\left\{x \in \operatorname{Spec}(A) \mid M_{x}\right.$ is not $A_{x}$-free $\}$.

(4) If hd ${ }_{A} M \leq 1$, then $M_{\#}$ is an invertible $A$-module, and $\operatorname{Supp}_{\#}^{t}=\operatorname{Sing}_{A}(M)$.

Let $M$ be a generically-free $A$-module of rank $d$. Choose a finite presentation

$$
0 \rightarrow N \rightarrow F \rightarrow M \rightarrow 0
$$

where $F$ is $A$-projective module of rank $m$, and we consider the chain complex $K(M)$ defined as follows (see [2]):

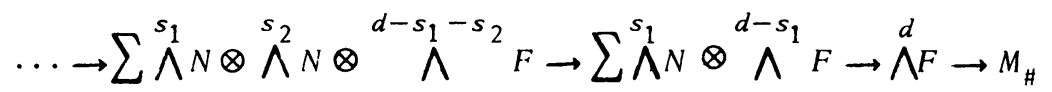

where $s_{i}>0$ for all $i$ (and hence it is a complex of length $d+1$ ), and the boundary operators are given by

$$
\begin{aligned}
u_{1} \otimes u_{2} \otimes \cdots \otimes u_{r} \otimes v \rightarrow & u_{1} \wedge u_{2} \otimes u_{3} \otimes \cdots \otimes u_{2} \otimes v \\
& -u_{1} \otimes u_{2} \wedge u_{3} \otimes \cdots \otimes u_{r} \otimes v \\
& +\cdots+(-1)^{2} u_{1} \otimes \cdots \otimes u_{r-1} \otimes u_{r} \wedge v .
\end{aligned}
$$

We note that $H_{0}(K(M))=M_{\#}^{t}$ and $H_{1}(K(M))=\operatorname{Ker}\left(\bigwedge^{d} M \rightarrow M_{\#}\right)=\left(\bigwedge^{d} M\right)_{t}$. Furthermore, if $M$ is $A$-projective (so that the inclusion map $N \rightarrow F$ admits a retract), then $K(M)$ is homotopically trivial, and in particular Supp $H_{*}(K(M)) \subset$ Sing ${ }_{A}(M)$. We state below the basic facts obtained in [2] concerning the complex $K(M)$. They are Theorem 2.4 and Corollary 4.4 in [2] respectively.

Lemma 1.1. Let $M$ be a generically-free A-module of finite type of rank $d$, with hd ${ }_{A} M \leq 1$. Then we have

(a) $d+1-\operatorname{Sing}(M)-\operatorname{depth} A=$ the smallest integer $q$ such that $H_{i}(K(M))=0$ for all $i>q$.

(b) Let $A$ be a local ring and assume that $M_{x}$ is $A_{x}$-free for all nonmaximal points $x$ in $\operatorname{Spec}(A)$. If $d+1>\operatorname{dim} A$, then $\chi\left(H_{*}(K(M))=0\right.$ where $\chi\left(H_{*}(K(M))\right)=$ $\sum_{i}(-1)^{i}$ length $H_{i}(K(M))$. 
As an immediate application, we obtain the following:

Theorem 1.2. Let $A$ be a commutative noetberian ring and $M$ a genericallyfree $A$-module of finite type with hd ${ }_{A} M \leq 1$. We set $d=\mathrm{rg}_{A} M$. Then

(i) If Sing (M) - depth $A \geq d$, then hd ${ }_{A} \wedge^{d} M \leq d$. If $\operatorname{Sing}(M)-\operatorname{depth} A>d$, then $\bigwedge^{d} M$ bas no torsion so that $0 \rightarrow \bigwedge^{d} M \rightarrow M_{\#} \rightarrow M_{\#}^{t} \rightarrow 0$ is exact, and $M_{\#}^{t}$ is a perfect (4) A-module with hd ${ }_{A} M_{\#}^{t}=d+1$.

(ii) Assume that $\operatorname{Sing}(M)$ is a finite set consisting of Coben-Macaulay maximal points of dimension $d$. We then bave length $\left(\left(\bigwedge^{d} M\right)_{t}\right)=$ length $\left(M_{\#}^{t}\right)$.

Proof. Choose a finite presentation of $M$

$$
0 \rightarrow N \rightarrow F \rightarrow M \rightarrow 0
$$

and consider the complex $K(M)$ as defined above.

(i) Suppose: that $\operatorname{Sing}(M)-\operatorname{depth} A \geq d$. By 1.1 (a), we then have $H_{i}(K(M))=0$ for all $i>1$, and therefore

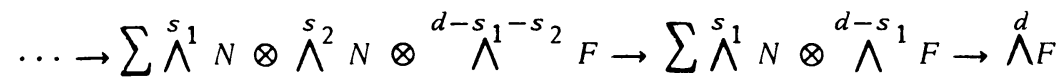

is an $A$-projective resolution of $\bigwedge^{d} M$, which shows that hd ${ }_{A} \bigwedge^{d} M \leq d$. If Sing $(M)-\operatorname{depth} A>d$, then $H_{i}(K(M))=0$ for all $i>0$ by 1.1 (a) again, and in particular we have $0=H_{1}(K(M))=\left(\bigwedge^{d} M\right)_{t}$.

(ii) Assume that $\operatorname{Sing}(M)$ is a finite set consisting of Cohen-Macaulay maximal points of dimension $d$. We then have

$$
\chi^{\left(H_{*}(K(M))\right)}=\sum_{x \in \operatorname{Sing}(M)} \chi^{\left(H_{*}\left(K\left(M_{x}\right)\right)\right)=0}
$$

by $1.1(\mathrm{~b})$. On the other hand, we have $H_{i}(K(M))=0$ for all $i>1$ by 1.1 (a) and therefore $0=\chi^{\left(H_{*}(K(M))\right)}=$ length $H_{0}(K(M))$ - length $H_{1}(K(M))=$ length $\left(M_{\#}^{t}\right)-$ length $\left(\left(\bigwedge^{d} M\right)_{t}\right)$.

Let $R$ be a noetherian ring and $A$ a generically smooth $R$-algebra of finite type (i.e. $A \otimes_{R} K$ is smooth over $K$ where $K$ is the total ring of fractions of $R$ ). Then the module of relative differentials $\Omega_{A \mid R}$ is a generically-free $A$-module of finite type such that $\operatorname{rg} \Omega_{A \mid R}=\operatorname{dim} A \otimes_{R} K$, and in turn we may consider $\wedge^{d} \Omega_{A \mid R} \rightarrow\left(\Omega_{A \mid R}\right)_{\#}$ where $d=\mathrm{rg} \Omega_{A \mid R}=\operatorname{dim} A \otimes_{R} K$. We note that in case when $A$ is a relative complete-intersection over $R$, then hd $\Omega_{A \mid R} \leq 1$ and $\left(\Omega_{A \mid R}\right)_{\#}$ is nothing but the module of dualizing differentials $\omega_{A \mid R}$ defined by A. Grothendieck [7], and the canonical map $\bigwedge^{d} \Omega_{A \mid R} \rightarrow \omega_{A \mid R}$ is an isomorphism at each point $x \in \operatorname{Spec}(A)$ at which $A$ is smooth over $R$, and therefore $\operatorname{Supp}\left(\left(\bigwedge^{d} \Omega_{A \mid R}\right)_{t}\right)$ as well as $\operatorname{Supp} \omega_{A \mid R}^{t}$ are contained in $\operatorname{Sing}(A \mid R)=\{x \in \operatorname{Spec}(A) \mid A$ is not smooth over $R$ at $x\}$. We note furthermore that $\operatorname{Sing}(A \mid R)=\operatorname{Sing} \Omega_{A \mid R}=\operatorname{Supp} \omega_{A \mid R}^{t}$ since $A$ is a relative complete-intersection over $R$, where $\omega_{A \mid R}^{t}=\operatorname{Coker}\left(\bigwedge^{d} \Omega_{A \mid R} \rightarrow \omega_{A \mid R}\right)$.

$\left(^{4}\right) A$-module $E$ of finite type is called perfect if hd $E<\infty$ and $\operatorname{Ext}_{A}^{i}(E, A)=0$ for all $i<$ hd ${ }_{A} E$ or equivalently $\operatorname{Supp}(E)-\operatorname{depth} A \geq \mathrm{hd}_{A} E$. 
Corollary 1.3. Let $R$ be Coben-Macaulay and $A$ a generically-smooth relative complete-intersection over $R$, of relative dimension $d$.

(i) If $\operatorname{dim} A_{x} \geq d$ for all $x \in \operatorname{Sing}(A \mid R)$, then $\operatorname{hd}_{A} \bigwedge^{d} \Omega_{A \mid R} \leq d$. If $\operatorname{dim} A_{x}>d$ for all $x \in \operatorname{Sing}(A \mid R)$, then $0 \rightarrow \bigwedge^{d} \Omega_{A \mid R} \rightarrow \omega_{A \mid R} \rightarrow \omega_{A \mid R}^{t} \rightarrow 0$ is exact.

(ii) Let $R$ be artinian. If $\operatorname{Sing}(A \mid R)$ consists of a finite number of maximal points, then we have length $\left(\left(\bigwedge^{d} A \mid R_{t}\right)\right)=\operatorname{length}\left(\omega_{A \mid R}^{t}\right)$.

Proof. Since $R$ is Cohen-Macaulay and $A$ is a relative complete-intersection over $R, A$ is also Cohen-Macaulay. Thus our statements follow immediately from 1.2 .

Before we consider a further application, we recall Fitting ideals of a module: Let $E$ be an $R$-module of finite type, and choose a finite presentation

$$
0 \rightarrow N \rightarrow F \rightarrow E \rightarrow 0
$$

where $F$ is $R$-free module of rank $n$. For each positive integer $p$, we set

$$
I_{R}^{(p)}(E)=\operatorname{Im}\left(\operatorname{det}: \bigwedge^{n-p+1} N \otimes \bigwedge^{n-p+1} F \rightarrow R\right)
$$

These ideals do not depend on the choice of a presentation of $E$ and hence are the invariants of $E$. For the sake of simplicity, we set $I_{R}(e)=I_{R}^{(1)}(E)$. We note that $\operatorname{Supp} R / I_{R}(E)=\operatorname{Supp} E$.

Lemma 1.4. Let $R$ be a local ring with the maximal ideal $\underline{m}$, and $A a$ (noetherian) $R$-flat algebra, sucb that $\underline{m} A$ is contained in the Jacobian radical of $A$. Then for any ideal $I$ in $A$, we bave

$$
I \text { - depth } A \geq(I \cap R)-\operatorname{depth} R+I-\operatorname{depth} R / m{\underset{R}{\otimes}}_{A} A .
$$

Proof. If $r_{1}, r_{2}, \cdots, r_{k}$ is an $R$-regular sequence in $I \cap R$, then it is an $A$ regular sequence since $A$ is $R$-flat. Therefore, replacing $R$ by $R /\left(r_{1}, \cdots, r_{s}\right)$ where $r_{1}, \cdots, r_{s}$ is a maximal $R$-regular sequence in $I \cap R$, we may assume that $(I \cap R)-\operatorname{depth} R=0$. Let $f$ be an element in $I$ which is not a zero-divisor in $R / \underline{m} \otimes_{R} A$. It suffices to show that $f$ is not a zero-divisor in $A$ and that $A / f A$ is $R$-flat. Consider the exact sequence $0 \rightarrow f A \rightarrow A \rightarrow A / f A \rightarrow 0$, which induces the exact sequence

$$
R / \underline{m} \underset{R}{\otimes} f A \rightarrow R / \underline{m} \underset{R}{\otimes} A \rightarrow R / \underline{m} \underset{R}{\otimes} A / f A \rightarrow 0 .
$$

Since the composite map $R / \underline{m} \otimes_{R} A \rightarrow{ }^{1 \otimes f} R / \underline{m} \otimes_{R} f A \rightarrow R / \underline{m} \otimes_{R} A$ is injection by hypothesis, and $R / \underline{m} \otimes_{R} A \rightarrow^{1 \otimes i} R / \underline{m} \otimes_{R} f A$ is surjection, it follows that $R / \underline{m} \otimes_{R} A \rightarrow 1 \otimes f R / \underline{m} \otimes_{R} f A$ is an isomorphism, and $R / \underline{m} \otimes f A \rightarrow R / \underline{m} \otimes_{R} A$ is an injection, i.e. $\operatorname{Tor}_{1}^{R}(R / \underline{m}, A / f A)=0$ which shows that $A / f A$ is again $A$-flat. 
Now consider the exact sequence $0 \rightarrow \operatorname{Ker} f \rightarrow A \rightarrow^{\prime} f A \rightarrow 0$. Since $A$ and $A / f A$ are $R$-flat, we have $\operatorname{Tor}_{1}^{R}(R / \underline{m}, f A)=0$ and hence we get the exact sequence

$$
0 \rightarrow R / \underline{m} \underset{R}{\otimes} \operatorname{Ker} f \rightarrow R / \underline{m} \underset{R}{\otimes} A \stackrel{1 \otimes f}{\longrightarrow} R / \underline{m} \underset{R}{\otimes} f A \rightarrow 0 .
$$

However, $R / \underline{m} \otimes_{R} A \rightarrow^{1 \otimes f} R / \underline{m} \otimes_{R} f A$ is an isomorphism and therefore $R / \underline{m} \otimes_{R}$ $\operatorname{Ker} f=0$. Since $\underline{m} A$ is contained in the Jacobson radical of $A$, it follows that $\operatorname{Ker} f=0$ by Nakayama's lemma.

Theorem 1.5. Let $R$ be a complete local ring with the maximal ideal $\underline{m}$, and $A$ a (noetherian) R-flat algebra. Let $M$ be a generically-free R-flat A-module of finite type with hd ${ }_{A} M \leq 1$. Assume that

(i) Sing $\left(M_{0}\right)$ consists of a finite number of Coben-Macaulay maximal points in Spec $\left(A_{0}\right)$ of dimension $\geq \mathrm{rg} M$, where $M_{0}=M / \underline{m} M$,

(ii) $K \otimes_{R} M$ is $K \otimes_{R}$ A-projective, where $K=$ the total ring of fractions of $R$,

(iii) $M_{\#}^{t}$ is of finite type as an $R$-module.

Then $\bigwedge^{d} M$ bas no torsion, $M_{\#}^{t}$ is a perfect $A$-module with $\mathrm{hd}_{A} M_{\#}^{t}=d+1$, and $I_{R}\left(M_{\#}^{t}\right)$ is an invertible ideal in $R$.

Proof. Set $I=\operatorname{Ann}_{A} M_{\#}^{t}$. Then (iii) entails that $A / I$ is of finite type as an $R$-module, i.e. $A / I$ is an integral $R$-algebra of finite type, and hence there are only a finite number of maximal ideals $\underline{m}_{1}, \underline{m}_{2}, \cdots, \underline{m}_{r}$ of $A$ containing $I$, and all of them dominate the maximal ideal $\underline{m}$ of $R$. Set $A^{\prime}=S^{-1} A$ where $S=A-$ $\underline{m}_{1} \cup \cdots \cup \underline{m}_{r}$, and set $M^{\prime}=A^{\prime} \otimes_{A} M$. Then $A^{\prime}$ is an $R$-flat algebra such that $\underline{m} A^{\prime}$ is contained in the Jacobson radical of $A^{\prime}$, and $M^{\prime}$ is a generically-free $R$-flat $A^{\prime}$-module of finite type with hd $A^{\prime} M^{\prime} \leq 1$, and the hypotheses (i), (ii), (iii) are carried over to $A^{\prime}$-module $M^{\prime}$. Furthermore, $A^{\prime} \otimes_{A} A / I=A / I$ entails that $\left(M^{\prime}\right)_{\#}^{t} \simeq A^{\prime} \otimes_{A} M_{\#}^{t} \simeq M_{\#}^{t}$ and hence $I_{R}\left(\left(M^{\prime}\right)_{\#}^{t}\right)=I_{R}\left(M_{\#}^{t}\right)$. Consequently, replacing $A$ by $A^{\prime}$, we may assume that $\underline{m} A$ is contained in the Jacobson radical of $A$. The hypothesis (ii) entails that Ass $R \cap \operatorname{Supp} R / I \cap R=\varnothing$ and hence $(I \cap R)-$ depth $R>0$. Therefore it follows from 1.4 that $\operatorname{Sing}(M)-\operatorname{depth} A>\operatorname{Sing}\left(M_{0}\right)-$ depth $A_{0}$. Since $\operatorname{Sing}\left(M_{0}\right)-\operatorname{depth} A_{0} \geq d(=\operatorname{rg} M)$ by hypothesis (i), we get $\operatorname{Sing}(M)$ - $\operatorname{depth} A>d$, and therefore it follows from 1.2 (i) that

$$
0 \rightarrow \stackrel{d}{\wedge} M \rightarrow M_{H} \rightarrow M_{H}^{t} \rightarrow 0
$$

is exact and $M_{\#}^{t}$ is a perfect $A$-module with hd ${ }_{A} \mathrm{M}_{\#}^{t}=d+1$. Since $M$ as well as $A$ are $R$-flat, so are $\bigwedge^{d} M$ and $M_{\#}$. Consequently, flat $-\operatorname{dim}_{R} M_{\#}^{t} \leq 1$, and hence $\mathrm{hd}_{R} M_{\#}^{t} \leq 1$ since $M_{\#}^{t}$ is an $R$-module of finite type. Let

$$
0 \rightarrow R^{m} \stackrel{b}{\longrightarrow} R^{i n} \longrightarrow M_{H}^{t} \longrightarrow 0
$$

be a finite presentation of $M_{\#}^{t}$ as an $R$-module. Since $M_{\#}^{t}$ is a torsion module by 
the hypothesis (ii), we must have $m=n$, and therefore $I_{R}\left(M_{\#}^{t}\right)=(\operatorname{det} b)$ and $\operatorname{det} b$ is not a zero-divisor in $R$.

Remark. If $A$ is complete under the $\underline{m}$-adic topology, then the hypothesis (iii) is a consequence of (i). Indeed, $M_{\#}^{t}$, being an $A$-module of finite type, is complete under the $\underline{m}$-adic topology and $R / \underline{m} \otimes_{R} M_{\#}^{t}$ is a finite-dimensional vector space over $R / \underline{m}$ by (i), and therefore must be of finite type as an $R$-module.

2. Torsion differentials and deformations. We briefly recall here the notion of a (formal) Schlessinger deformation: Let $k$ be a fixed perfect field, and $X_{0}$ a scheme over $k$. A deformation of $X_{0}$ is meant by a pair $(R, X)$ where $R$ is a complete noetherian local $k$-algebra with the residue field $k$, and $X$ a flat $R$ scheme together with a Cartesian diagram:

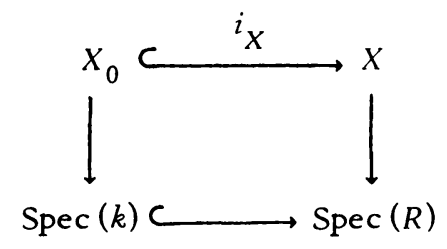

A morphism $(R, X) \rightarrow\left(R^{\prime}, X^{\prime}\right)$ of deformations of $X_{0}$ is a pair $(b, \phi)$ where $b$ : $R \rightarrow R^{\prime}$ is a local $k$-algebra map and $\phi: X^{\prime} \rightarrow X$ is a morphism of schemes with a commutative diagram:

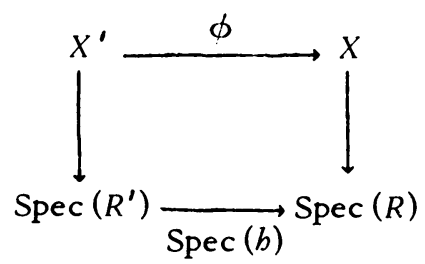

A formal deformation of $X_{0}$ is a pair $(R, X)$ where $R$ is a complete noetherian local $k$-algebra with the maximal ideal $\underline{m}$, and $X$ a $R$-flat $\underline{m}$-adic formal scheme together with a Cartesian diagram

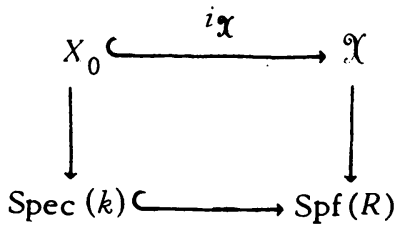

in the category of formal schemes. Thus if we set by $\underline{M}_{X_{0}} \cdot \hat{M}_{X_{0}}$ the category of deformations and formal deformations of $X_{0}$ respectively, then we get a canonical functor $\sim: \underline{M}_{X_{0}} \rightarrow \underline{\hat{M}}_{X_{0}}$ via $(R, X) \rightarrow(R, \hat{X})$ where $\hat{X}$ denotes the formal $\underline{m}$-adic completion of $X$. We note that if $X_{0}$ is proper over $k$ then the functor - is a full-faithful imbedding, and that $a$ is an equivalence of categories in case when $X_{0}$ is a projec- 
tive variety with $H^{2}\left(X_{0}, \underline{O}_{X_{0}}\right)=0$ (for instance the case when $X_{0}$ is a complete curve) [5].

For each complete noetherian local $k$-algebra $S$, we set $\hat{M}_{X}(S)=$ the category of formal deformations of $X_{0}$ over $S$, the morphisms being the one which is the identity on $S$. We may note that every morphism in the category $\underline{\underline{M}}_{X}(S)$ is necessarily an isomorphism, i.e. $\hat{\underline{M}}_{X}(S)$ is a groupoid. Let $(R, X)$ be a fixed formal deformation of $X_{0}$. For any local map $R \rightarrow R^{\prime},\left(R^{\prime}, X \hat{\otimes}_{R} R^{\prime}\right)$ is a formal deformation of $X_{0}$ over $R^{\prime}$, and therefore $(R, \Upsilon)$ induces a canonical map

$$
\left(\widetilde{R, X)}: \operatorname{Hom}_{\text {local } k \text {-alg }}\left(R, R^{\prime}\right) \rightarrow\left[\underline{\hat{M}}_{X_{0}}\left(R^{\prime}\right)\right]\right.
$$

where $\left[\underline{M}_{X}{ }_{0}\left(R^{\prime}\right)\right]$ denotes the set of isomorphism classes of objects in $\hat{M}_{X_{0}}\left(R^{\prime}\right)$.

Definition 2.1(5). A (formal) deformation $(R, X)$ of $X_{0}$ is called a versal (formal) deformation of $X_{0}$ if every (formal) deformation of $X_{0}$ is induced from $(R, X)$. In other words, a versal deformation or a versal formal deformation of $X_{0}$ is a quasi-initial object in the category $\underline{M}_{X}, \underline{\hat{M}}_{X_{0}}$ respectively. A versal (formal) deformation $(R, X)$ such that

$$
(R, X): \operatorname{Hom}_{\text {localk-alg }}(R, k[\epsilon]) \rightarrow\left[\underline{M}_{X_{0}}(k[\epsilon])\right]
$$

is a bijection is called a minimally versal (formal) deformation or a Schlessinger (formal) deformation of $X_{0}$.

Any two (formal) Schlessinger deformations of $X_{0}$ are easily seen to be (noncanonically) isomorphic. A basic theorem on deformations is the following existence theorem due to $M$. Schlessinger [10] supplemented by a theorem of $A$. Grothendieck [5].

Theorem 2.2. Let $X_{0}$ be a scheme over $k$. If Sing $\left(X_{0}\right)=\left\{x \in X_{0} \mid X_{0}\right.$ is not smooth over $k$ at $x\}$ is proper over $k$, then there exists a formal Schlessinger deformation $(R, X)$ of $X_{0}$. If, furthermore, $X_{0}$ is projective over $k$ and $H^{2}\left(X_{0}, O_{X_{0}}\right)=$ 0 , then there exists a Schlessinger deformation of $X_{0}$.

Definition 2.3. Let $(R, X)$ be a formal Schlessinger deformation of $X_{0}$. We set $s_{X_{0}}=\operatorname{dim} R$. It is an invariant of $X_{0}$.

Remark 2.4. Let $X_{0}$ be an affine scheme over $k$, and set $\Gamma\left(X_{0}, \underline{O}_{X_{0}}\right)=A_{0}$. We then have a canonical isomorphism $\left[\underline{M}_{X_{0}}(k[\epsilon])\right] \simeq D^{1}\left(A_{0} \mid k, A_{0}\right)$ via

$$
(k[\epsilon], \operatorname{Spec}(A)) \rightarrow\left(0 \rightarrow A_{0} \stackrel{\epsilon}{\longrightarrow} A \rightarrow A_{0} \rightarrow 0\right)
$$

where $D^{1}\left(A_{0} \mid k, A_{0}\right)=$ the isomorphism classes of commutative $k$-algebra exten-

(5) See footnote (3). 
sions of $A_{0}$ by $A_{0}$. Now assume that $X_{0}$ is a complete-intersection over $k$ of dimension $r$ with isolated nonsmooth points only, and choose a presentation

$$
0 \rightarrow I \rightarrow P \rightarrow A_{0} \rightarrow 0
$$

where $P=k\left[x_{1}, \cdots, x_{n}\right]$, and $I=\left(f_{1}, f_{2}, \cdots, f_{n-r}\right)$ is generated by a $P$-regular sequence. We then have the exact sequence

$$
\operatorname{Der}_{k}\left(P, A_{0}\right) \rightarrow \operatorname{Hom}_{A_{0}}\left(I / I^{2}, A_{0}\right) \rightarrow D^{1}\left(A_{0} \mid k, A_{0}\right) \rightarrow 0
$$

and $D^{1}\left(A_{0} \mid k, A_{0}\right)$ is a finite-dimensional vector space over $k$ since $A_{0}$ has isolated nonsmooth points only. We set $s=\operatorname{dim}_{k} D^{1}\left(A_{0} \mid k, A_{0}\right)$, and choose $\phi_{i}: I / I^{2}$ $\rightarrow A_{0}(i=1,2, \cdots, s)$ representing $k$-basis elements of $D^{1}\left(A_{0} \mid k, A_{0}\right)$, and then choose $M_{i j}$ in $P$ such that $\phi_{i}\left(f_{j}\right)=M_{i j}(\bmod I)$. If we set $R=k\left[\left[t_{1}, \cdots, t_{s}\right]\right]$ and $A=R\left\{X_{1}, \cdots, X_{n}\right\} /\left(F_{1}, F_{2}, \cdots, F_{n-r}\right)$ where

$$
\left\{\begin{array}{l}
F_{i}=f_{i}+\sum_{j=1}^{s} t_{j} M_{i j} \\
R\left\{X_{1}, \cdots, X_{n}\right\}=\text { the restricted formal power-series ring over the adic-ring } R
\end{array}\right.
$$

then $A$ is $R$-flat, and indeed $(R, \operatorname{Spf}(A))$ is a formal Schlessinger deformation of $X_{0}$ (see [8] for its detail). In particular, we have $s_{X_{0}}=\operatorname{dim}_{k} D^{1}\left(A_{0} \mid k, A_{0}\right)$. We note that $F_{1}, F_{2}, \cdots, F_{n-r}$ are all polynomials with coefficients in $R$ and hence Spf $(A)$ is "algebraic", i. e. Spf $(A)=\widehat{\operatorname{Spec}(B)}$ where $B=$ $R\left[X_{1}, \cdots, X_{n}\right] /\left(F_{1}, \cdots, F_{n-r}\right)$. Now assume that $A_{0}$ is reduced so that $A_{0}$ is, since $k$ is a perfect field, generically smooth over $k$. If we set $N=$ $\operatorname{Im}\left(I / I^{2} \rightarrow^{d} A_{0} \otimes_{P} \Omega_{P \mid k}\right)$, then $I / I^{2} \rightarrow^{d} N$ is an isomorphism at each generic point of Spec $\left(A_{0}\right)$, and therefore Supp $(\operatorname{Ker} d)$ contains none of the generic points of $\operatorname{Spec}\left(A_{0}\right)$, and consequently $\operatorname{Hom}_{A_{0}}\left(N, A_{0}\right) \rightarrow \operatorname{Hom}_{A_{0}}\left(I / I^{2}, A_{0}\right)$ is an isomorphism. Then the exact sequence

$$
0 \rightarrow N \rightarrow A_{0} \underset{P}{\otimes} \Omega_{P \mid k} \rightarrow \Omega_{A_{0} \mid k} \rightarrow 0
$$

together with the fact that $A_{0} \otimes_{P} \Omega_{P \mid k}$ is $A_{0}$-free module entails that $\operatorname{Ext}_{A_{0}}^{1}\left(\Omega_{A_{0} \mid k}, A_{0}\right) \rightarrow D^{1}\left(A_{0} \mid k, A_{0}\right)$ is an isomorphism. We thus conclude that if $X_{0}=\operatorname{Spec}\left(A_{0}\right)$ is a reduced complete-intersection over $k$ with isolated nonsmooth points only, then $s_{X_{0}}=\operatorname{dim}_{k} \operatorname{Ext}_{A_{0}}^{1}\left(\Omega_{A_{0} \mid k}, A_{0}\right)$.

Remark 2.5. Let $X_{0}=\operatorname{Spec}\left(A_{0}\right)$ be a complete-intersection over $k$. Then we set $A_{0}=k\left[x_{1}, x_{2}, \ldots, x_{n}\right] /\left(f_{1}, f_{2}, \ldots, f_{r}\right)$ where $f_{1}, f_{2}, \ldots, f_{r}$ is a $k\left[x_{1}, \cdots, x_{n}\right]$-regular sequence. Then $X_{0}$ admits a generically-smooth deformation, i.e. $X_{0}$ can be "desingularized via deformation". Indeed, set $S=k[[t]]$ and $A=S\left[X_{1}, \ldots, X_{n}\right] /\left(F_{1}, \cdots, F_{r}\right)$ where $F_{j}=(1-t) f_{j}+t X_{j}$ for $j=1,2$, $\cdots, r$. Since $F_{j} \equiv f_{j}(\bmod t A)$ and $f_{1}, f_{2}, \cdots, f_{r}$ is an $A / t A$-regular sequence, 
it follows that $A$ is $S$-flat, i.e. $(S, \operatorname{Spec}(A))$ is a deformation of $X_{0}$ over $S$. Now $A=S \otimes_{k[t]} A^{\prime}$, where $A^{\prime}=k\left[t, X_{1}, \ldots, X_{n}\right] /\left(F_{1}, \ldots, F_{r}\right)$, and $A^{\prime} /(t-1) A^{\prime} \simeq$ $k\left[X_{1}, \ldots, X_{n}\right] /\left(X_{1}, \cdots, X_{r}\right) \simeq k\left[X_{r+1}, \cdots, X_{n}\right]$. Therefore, $k(t) \otimes_{k}[t] A^{\prime}$ is smooth over $k(t)$ and hence $k((t)) \otimes_{k}[[t]]$ is smooth over $k((t))$, i.e. $(S, \operatorname{Spec}(A))$ is a generically-smooth deformation of $X_{0}$.

In this section we are interested in the deformation of complete curves. For this purpose we need the following lemma.

Lemma 2.6. Let $X_{0}$ be a k-scheme of finite type with isolated nonsmooth points only. Assume that $H^{2}\left(X_{0}, \underline{\Omega}_{X}^{*}\right)=0$ where

$$
\underline{\Omega}_{X}^{*}=\underline{\operatorname{Hom}}_{\underline{O}_{X_{0}}}\left(\underline{\Omega}_{X_{0}}, \underline{O}_{X_{0}}\right) .
$$

Then

(i) $s_{X_{0}}=\operatorname{dim}_{k} H^{1}\left(X_{0}, \underline{\Omega}_{X_{0}}^{*}\right)+s_{U_{0}}$ where $U_{0}$ is any affine open subscheme of $X_{0}$ containing Sing $\left(X_{0} \mid k\right)=\left\{x \in X_{0} \mid X_{0}\right.$ is not smooth over $k$ at $\left.x\right\}$.

(ii) Let $(R, X)$ be a formal Schlessinger deformation of $X_{0}$. Then for any affine open $V_{0} \subset X_{0},\left(R, X \mid V_{0}\right)$ is a versal formal deformation of $V_{0}$.

The above lemma is not difficult to prove, the main reason being that $H^{2}\left(X_{0}, \underline{\Omega}_{X_{0}}^{*}\right)=0$ entails the vanishing of the global obstructions. In any case, a detailed argument can be found in [8] and thus we omit its proof here. Let $X_{0}$ be a complete curve over $k$, and let $(R, X)$ be a Schlessinger deformation of $X_{0}$. We note that the functor $\leadsto: \underline{M}_{X_{0}} \rightarrow \underline{M}_{X_{0}}$ is an equivalence of categories since $X_{0}$ is projective over $k$ and $H^{2}\left(X_{0}, \underline{O}_{X_{0}}\right)=0$, and therefore a Schlessinger deformation of $X_{0}$ does exist. Assume that $X_{0}$ is a relative complete intersection over $k$. Then $X$, being $R$-flat, is a relative complete-intersection over $R$ and hence Sing $(X \mid R)=\operatorname{Supp} \omega_{X \mid R}^{t}$ where Sing $(X \mid R)=\{x \in X \mid X$ is not smooth over $R$ at $x\}$, $\omega_{X \mid R}^{t}=\operatorname{Coker}\left(\underline{\Omega}_{X \mid R} \rightarrow \omega_{X \mid R}\right)$, and $\omega_{X \mid R}$ is the dualizing sheaf of A. Crothendieck [7]. In view of this, we may provide the closed subset $\operatorname{Sing}(X \mid R)$ with the closed subscheme structure defined by the ideal sheaf $\underline{I}_{X}\left(\omega_{X}^{t}\right)$, where $\underline{I}_{X}(\underline{E})$, for any coherent $X$-module $\underline{E}$, stands for the ideal sheaf of $\underline{O}_{X}$ given locally by the 1 st Fitting ideal of $\underline{E}$, i.e. for each affine open $U=\operatorname{Spec}(A) \subset X$,

$$
\Gamma\left(U, \underline{I}_{X}(\underline{E})\right)=\operatorname{Im}\left(\operatorname{det}: \bigwedge^{m} N \otimes \bigwedge^{m} F \rightarrow A\right)
$$

where $0 \rightarrow N \rightarrow F \rightarrow \Gamma^{\top}(U, \underline{E}) \rightarrow 0$ is a finite presentation of $A$-module $\Gamma(U, \underline{E})$ in which $F$ is $A$-free, and $m$ is the free rank of $A$-module $F$. We note that, for any coherent $X$-module $E$, the ideal sheaf $\underline{I}_{X}(\underline{E})$ annihilates $\underline{E}$, and in particular $\underline{\omega}_{X}^{t}$ is a coherent Sing $(X \mid R)$-module. We are also interested in "the image of Sing $(X \mid R)$ under $f$ ". Since $f: X \rightarrow \operatorname{Spec}(R)$ is proper, $\Gamma\left(X, \omega_{X \mid R}^{t}\right)$ is an $R$-module of finite type and $\operatorname{Supp} \Gamma\left(X, \omega_{X \mid R}^{t}\right)=f(\operatorname{Sing}(X \mid R))$. We thus note that $f(\operatorname{Sing}(X \mid R))$ 
$=\operatorname{Supp} R / I_{R}\left(I\left(X,\left.\omega_{X}^{t}\right|_{R}\right)\right)$ where $I_{R}\left(\Gamma\left(X, \omega_{X \mid R}^{t}\right)\right)$ is the 1st Fitting ideal of the $R$ module $\Gamma\left(X, \omega_{X \mid R}^{t}\right)$. The main purpose of this section is to establish the following.

Theorem 2.7( $\left.{ }^{6}\right)$. Let $X_{0}$ be a reduced complete curve defined over $k$, and let $(R, X)$ be a Schlessinger deformation of $X_{0}$. Assume that $X_{0}$ is a relative complete intersection over $k$. We then bave

(i) $s_{X_{0}}=3 g-3+\operatorname{dim}_{k} H^{0}\left(X_{0}, \underline{\Omega}_{X_{0}}^{*}\right)$ where $g=\operatorname{dim}_{k} H^{1}\left(X_{0}, \underline{O}_{X_{0}}\right)$.

(ii) Sing $(X \mid R) \rightarrow \operatorname{Spec}(R)$ is a finite morphism, and Sing $(X \mid R)=\operatorname{Sing}_{x_{1}}(X \mid R)$ $\cup \ldots \cup \operatorname{Sing}_{x_{m}}(X \mid R)$ is a disjoint union, where $\left\{x_{1}, x_{2}, \ldots, x_{m}\right\}=\operatorname{Sing}\left(X_{0} \mid k\right)$, and Sing $_{x}(X \mid R)$ stands for the connected component of Sing $(X \mid R)$ containing the point $x$.

(iii) $\Omega_{X \mid R}$ bas no torsion, the canonical map $\omega_{X \mid R}^{*} \rightarrow \Omega_{X \mid R}^{*}$ is an isomorphism, and $I_{R}\left(\Gamma\left(X, \omega_{X \mid R}^{t}\right)\right)$ is an invertible ideal in $R$. In particular, $\Omega_{X \mid R}^{*}$ is an invertible sheaf and $\operatorname{codim}(f(\operatorname{Sing}(X \mid R))$ in $\operatorname{Spec}(R))=1$.

Proof. (i) Let $U_{0}=\operatorname{Spec}\left(A_{0}\right)$ be an affine open subscheme of $X_{0}$ containing Sing $\left(X_{0} \mid k\right)$. Since $X_{0}$ is a relative complete-intersection over $k$, we may assume that $A_{0}=k\left[x_{1}, x_{2}, \cdots, x_{n+1}\right] /\left(f_{1}, f_{2}, \cdots, f_{n}\right)$ where $f_{1}, f_{2}, \ldots, f_{n}$ is a $k\left[x_{1}, \ldots, x_{n+1}\right]$-regular sequence. Since $A_{0}$ is reduced by hypothesis, we have

$$
s_{U_{0}}=\operatorname{dim}_{k} \operatorname{Ext}_{A_{0}}^{1}\left(\Omega_{A_{0} \mid k}, A_{0}\right)=\operatorname{dim}_{k} H^{0}\left(U_{0}, \underline{\operatorname{Ext}}_{X_{0}}^{1}\left(\underline{\Omega}_{X_{0} \mid k}, \underline{O}_{X_{0}}\right)\right)
$$

by 2.4. However, Sing $\left(X_{0} \mid k\right) \subset U_{0}$ and hence Supp $\underline{\operatorname{Ext}}_{X_{0}}^{1}\left(\underline{\Omega}_{X_{0}} \mid k, \underline{O}_{X_{0}}\right) \subset U_{0}$ and therefore

$$
H^{0}\left(X_{0}, \underline{\operatorname{Ext}}_{\mathrm{x}_{0}}^{1}\left(\underline{\Omega}_{X_{0} \mid k}, \underline{O}_{X_{0}}\right)\right)=H^{0}\left(U_{0}, \underline{\operatorname{Ext}}_{X_{0}}^{1}\left(\underline{\Omega}_{X_{0}} \mid k, \underline{O}_{X_{0}}\right)\right) .
$$

It follows from 2.6 that

$$
s_{X_{0}}=\operatorname{dim}_{k} H^{1}\left(X_{0}, \underline{\Omega}_{X_{0} \mid k}^{*}\right)+\operatorname{dim}_{k} H^{0}\left(X_{0}, \underline{\operatorname{Ext}}_{X_{0}}^{1}\left(\underline{\Omega}_{X_{0} \mid k}, \underline{O}_{X_{0}}\right)\right) .
$$

Thus it suffices to show that

$\operatorname{dim} H^{1}\left(X_{0}, \underline{\Omega}_{X_{0} \mid k}^{*}\right)+\operatorname{dim} H^{0}\left(X_{0}, \underline{\operatorname{Ext}}_{X_{0}}^{1}\left(\underline{\Omega}_{X_{0} \mid k}, \underline{O}_{X_{0}}\right)\right)=3 g-3+\operatorname{dim} H^{0}\left(X_{0}, \underline{\Omega}_{X_{0} \mid k}^{*}\right)$

i.e. $\chi\left(\underline{\Omega}_{X_{0} \mid k}^{*}\right)=3-3 g+\operatorname{dim} H^{0}\left(X_{0}, \underline{\operatorname{Ext}}_{X_{0}}^{1}\left(\underline{\Omega}_{X_{0} \mid k}, \underline{O}_{X_{0}}\right)\right)$ where $\chi(\underline{E})=$ $\sum_{i}(-1)^{i} \operatorname{dim} H^{i}\left(X_{0}, \underline{E}\right)$ for any coherent $X_{0}$-module $\underline{E}$. Now consider the exact sequence

$$
0 \rightarrow\left(\underline{\Omega}_{X_{0}} \mid k\right)_{t} \rightarrow \underline{\Omega}_{X_{0} \mid k} \stackrel{i}{\rightarrow} \omega_{X_{0} \mid k} \rightarrow \omega_{X_{0} \mid k}^{t} \rightarrow 0
$$

Since

(6) P. Deligne has proven recently a stronger version of 2.7(i). He has communicated to me that the formula $2.7(i)$ is valid for any curve which is a specialization of a nonsingular curve. 


$$
\underline{H}^{\text {om }} \underline{O}_{X_{0}}\left(\left(\underline{\Omega}_{X_{0}} \mid k\right), \underline{O}_{X_{0}}\right)=0
$$

we get an isomorphism $i\left(\underline{\Omega}_{X_{0} \mid k}\right)^{*} \simeq \underline{\Omega}_{X_{0}}^{*}$. Since $X_{0}$ is a Gorenstein scheme, $\omega_{X_{0} \mid k}$ is an invertible $X_{0}$-module and it follows immediately $\underline{\operatorname{Ext}}_{X}^{i}\left(i\left(\underline{\Omega}_{X_{0}} \mid k\right), \underline{O}_{X_{0}}\right)=0$ for all $i>0$. Consequently we get

$$
\begin{gathered}
\underline{\operatorname{Ext}}_{X_{0}}^{1}\left(\underline{\Omega}_{X_{0} \mid k}, \underline{O}_{X_{0}}\right) \simeq \underline{\operatorname{Ext}}_{X_{0}}^{1}\left(\left(\underline{\Omega}_{X_{0}} \mid k\right), \underline{O}_{X_{0}}\right), \\
0 \rightarrow \omega_{X_{0} \mid k}^{*} \rightarrow \underline{\Omega}_{X_{0} \mid k}^{*} \rightarrow \underline{\operatorname{Ext}}_{X_{0}}^{1}\left(\omega_{X_{0} \mid k}^{t}, \underline{O}_{X_{0}}\right) \rightarrow 0 .
\end{gathered}
$$

The exact sequence $(* *)$ entails

$$
\begin{aligned}
\chi\left(\underline{\Omega}_{X_{0} \mid k}^{*}\right) & =\chi\left(\omega_{X_{0} \mid k}^{*}\right)+\operatorname{dim} H^{0}\left(X_{0}, \underline{\operatorname{Ext}}_{X_{0}}^{1}\left(\omega_{X_{0} \mid k}^{t}, \underline{O}_{X_{0}}\right)\right) \\
& =3-3 g+\operatorname{dim} H^{0}\left(X_{0}, \underline{\operatorname{Ext}}_{X_{0}}^{1}\left(\omega_{X_{0} \mid k}^{t}, \underline{O}_{X_{0}}\right)\right)
\end{aligned}
$$

On the other hand, the local duality over Gorenstein scheme together with the isomorphic $(*)$ entails

$$
\begin{aligned}
& \operatorname{dim} H^{0}\left(X_{0}, \underline{\operatorname{Ext}}_{X_{0}}^{1}\left(\omega_{X_{0} \mid k}^{t}, \underline{O}_{X_{0}}\right)\right)=\operatorname{dim} H^{0}\left(X_{0}, \omega_{X_{0} \mid k}^{t}\right), \\
& \operatorname{dim} H^{0}\left(X_{0}, \underline{\operatorname{Ext}}_{X_{0}}^{1}\left(\underline{\Omega}_{X_{0}} \mid k, \underline{O}_{X_{0}}\right)\right) \\
& \quad=\operatorname{dim} H^{0}\left(X_{0}, \underline{\operatorname{Ext}}_{X_{0}}^{1}\left(\left(\underline{\Omega}_{X_{0} \mid k}\right)_{t}, \underline{O}_{X_{0}}\right)\right)=\operatorname{dim} H^{0}\left(X_{0},\left(\underline{\Omega}_{X_{0} \mid k}\right)_{t}\right) .
\end{aligned}
$$

However, $X_{0}$ is a relative complete-intersection over $k$ and hence it follows from 1.3(ii) that $\operatorname{dim} H^{0}\left(X_{0},\left(\underline{\Omega}_{X_{0} \mid k}\right)_{t}\right)=\operatorname{dim} H^{0}\left(X_{0}, \underline{\omega}_{X_{0} \mid k}^{t}\right)$. Consequently we obtain that

$$
\left.\operatorname{dim} H^{0}\left(X_{0}, \underline{\operatorname{Ext}}_{X_{0}}^{1} \underline{\Omega}_{X_{0} \mid k}, \underline{O}_{X_{0}}\right)\right)=\operatorname{dim} H^{0}\left(X_{0}, \underline{\operatorname{Ext}}_{X_{0}}^{1}\left(\omega_{X_{0}}^{t} \mid k, \underline{O}_{X_{0}}\right)\right)
$$

and hence $\chi\left(\underline{\Omega}_{X_{0} \mid k}^{*}\right)=3-3 g+\operatorname{dim} H^{0}\left(X_{0}, \underline{\operatorname{Ext}} \underline{X}_{0}^{1}\left(\underline{\Omega}_{X_{0} \mid k}^{1}, \underline{O}_{X_{0}}\right)\right)$.

(ii) Sing $(X \mid R)$ being a closed subscheme of $X$, Sing $(X \mid R) \rightarrow \operatorname{Spec}(R)$ is proper. Since Sing $(X \mid R) \cap X_{0}=\operatorname{Sing}\left(X_{0} \mid k\right)$ is a finite set, i.e. $k \otimes_{R} \operatorname{Sing}(X \mid R)$ consists of a finite number of points, it follows that $\operatorname{Sing}(X \mid R) \rightarrow \operatorname{Spec}(R)$ is a finite morphism, and in particular Sing $(X \mid R)$ is affine. Set $\operatorname{Sing}(X \mid R)=\operatorname{Spec}\left(R^{\prime}\right)$. Since $R$ is a complete local ring and $R^{\prime}$ is integral over $R$ of finite type, we conclude that $R^{\prime}$ is a finite direct product of local rings, i.e. $R^{\prime}=R_{1} \times \cdots \times R_{r}$, and therefore Sing $(X \mid R)=V_{1} \cup \ldots \cup V_{r}$ (disjoint union) where $V_{i}=\operatorname{Spec}\left(R_{i}\right)$ and $R_{i}$ is a local ring. Since the underlying space of $k \otimes_{R} \operatorname{Sing}(X \mid R)$ is $\operatorname{Sing}\left(X_{0} \mid k\right)=$ $\left\{x_{1}, x_{2}, \cdots, x_{m}\right\}$, we conclude that $r=m$ and $V_{i}=\operatorname{Sing}_{x_{i}}(X \mid R)$.

(iii) Since $X_{0}$ is a relative complete-intersection over $k$ and $X$ is $R$-flat, it 
follows that $X$ is a relative complete-intersection over $R$. Since $X_{0}$ is 1-dimensional, we have $H^{2}\left(X_{0}, \underline{\Omega}_{X}^{*}\right)=0$ and therefore, for each affine open $U_{0} \subset X_{0}$, $\hat{X} \mid U_{0}$ is a versal formal deformation of $U_{0}$. On the other hand, since $U_{0}$ is a relative complete-intersection over $k, U_{0}$ admits a generically smooth deformation by 2.5 , and consequently we conclude that a Schlessinger deformation $X$ is generically smooth over $R$ since $R$ is a formal power-series ring so that $\operatorname{Spec}(R)$ is irreducible. Since Sing $\left(X_{0} \mid k\right)$ is a finite set, we may choose an affine open $U C$ $X$ containing $\operatorname{Sing}\left(X_{0} \mid k\right)$. Let $y \in \operatorname{Sing}(X \mid R)$. Since $\{\bar{y}\} \cap \operatorname{Sing}\left(X_{0} \mid k\right) \neq \varnothing$ so that $\{\bar{y}\} \cap U \neq \varnothing$, we find that $y \in U$. Therefore Sing $(X \mid R) \subset U$, i.e. Supp $\omega_{X \mid R}^{t} \subset U$. Consequently we have that $\Gamma\left(X, \omega_{X \mid R}^{t}\right)=\Gamma\left(U, \omega_{X \mid R}^{t}\right)$ and therefore we may replace $X$ by an affine open $U=\operatorname{Spec}(A)$ containing $\operatorname{Sing}\left(X_{0} \mid k\right)$. Then $A$-module $\Omega_{A \mid R}$ has the following properties:

(a) Sing $\left(k \otimes_{R} \Omega_{A \mid R}\right)=\operatorname{Sing}\left(\Omega_{A_{0} \mid k}\right)=\operatorname{Sing}\left(X_{0} \mid k\right)$ consists of a finite number of Cohen-Macaulay maximal points in $\operatorname{Spec}\left(k \otimes_{R} A\right)=\operatorname{Spec}\left(A_{0}\right)$ of dimension $\geq 1$ $=\operatorname{rg} \Omega_{A \mid R}$.

(b) $\Omega_{A \mid R}$ is $R$-flat (since $X$ is $R$-flat and is a relative complete-intersection over $R$ ), and $K \otimes_{R} \Omega_{A \mid R}$ is $K \otimes_{R} A$-projective (since $X$ is generically smooth over $R$ ).

(c) $\omega_{A \mid R}^{t}\left(=\Gamma\left(U, \omega_{X \mid R}^{t}\right)=\Gamma\left(X, \omega_{X \mid R}^{t}\right)\right)$ is an $R$-module of finite type.

It follows from 1.5 that $\Omega_{A \mid R}$ has no torsion, $\omega_{A \mid R}^{t}$ is a perfect $A$-module with hd $\omega_{A \mid R}^{t}=2$, and $I_{R}\left(\Gamma\left(X, \omega_{X \mid R}^{t}\right)\right)=I_{R}\left(\omega_{A \mid R}^{t}\right)$ is an invertible ideal in $R$. Since Sing $(X \mid R) \subset U$ and $\Omega_{A \mid R}=\Gamma\left(U, \Omega_{X \mid R}\right)$ has no torsion, it follows that $\Omega_{X \mid R}$ has no torsion, i.e.

$$
0 \rightarrow \Omega_{X \mid R} \rightarrow \omega_{X \mid R} \rightarrow \omega_{X \mid R}^{t} \rightarrow 0
$$

is exact. Since depth $\omega_{A \mid R}^{t}=2$, we have $\operatorname{Ext}_{X}^{1}\left(\omega_{X \mid R}^{t}, \underline{O}_{X}\right)=0$ for $i=0,1$, and therefore $\omega_{X \mid R}^{*} \rightarrow \Omega_{X \mid R}^{*}$ is an isomorphism. This completes our proof.

Remark. For any reduced complete curve $X_{0}$, we define $l_{X_{0}}=s_{X_{0}}$ $\operatorname{dim} H^{1}\left(X_{0},\left.\underline{\Omega}_{X}^{*}\right|_{k}\right)$ which measures the local contribution to the deformations of $X_{0}$. We note, as a consequence of $2.7(\mathrm{i})$, that if $X_{0}$ is a relative complete-intersection over $k$, then $l_{X_{0}}=\operatorname{dim} H^{0}\left(X_{0},\left(\Omega_{X_{0} \mid k}\right)_{t}\right)=\operatorname{dim} H^{0}\left(X_{0}, \omega_{X_{0} \mid k}^{t}\right)$.

3. Local connection and rigid singularities. Let $k$ be a fixed perfect field as before, and $X_{0}$ a reduced $k$-scheme.

Definition 3.1. $X_{0}$ is said to be a "limit of smooth $k$-schemes" or $X_{0}$ is a specialization of a smooth $k$-scheme if there exists a deformation $(R, X)$ of $X_{0}$ such that $X \otimes_{R} K$ is smooth over $K$, where $K$ is the total ring of fractions of $R$. We say that $X_{0}$ is rigid if every deformation of $X_{0}$ over $k[\epsilon]$ is trivial, i.e. for any deformation $(k[\epsilon], X)$ of $X_{0}$ we have $X \simeq X_{0} \otimes_{k} k[\epsilon]$. We note that if $X_{0}=$ $\operatorname{Spec}\left(A_{0}\right)$ is a reduced affine $k$-scheme, $X_{0}$ is rigid if and only if $\operatorname{Ext}_{A_{0}}^{1}\left(\Omega_{A_{0} \mid k}, A_{0}\right)$ 
$=0$ (see 2.4). We also note that a rigid $k$-scheme $X_{0}$ can never be a specialization of a smooth $k$-scheme unless $X_{0}$ itself is smooth over $k$.

Every reduced curve is conjecturally a specialization of a smooth curve [3].

We have also seen in 2.5 that every affine $k$-scheme which is a complete-intersection is a specialization of a smooth $k$-scheme. On the other hand, there exists a 4-dimensional affine variety which can never be a specialization of a smooth variety ([4], [11]). The main purpose of this section is to provide an example of rigid 2-dimensional singularity. To clarify our motivation, we consider the phenomena of local connection under specialization, for which the author is indebted to A. Grothendieck.

For any local ring $A$, we denote by Spex $(A)$ the open subscheme of Spec $(A)$ deleting the maximal point. A variance of the following lemma is contained in [6]. However, we give a complete proof for the convenience of the readers.

Lemma 3.2. Let $A$ be a noetherian local ring with the maximal ideal $\underline{m}$. If $\operatorname{depth} A \geq 2$ and $H_{\underline{m}}^{2}(A)$ is coberent, then for any A-regular element $t \in \underline{m}$, Spex $(A / t A)$ is connected.

Proof. Since $\operatorname{depth} A \geq 2$, we have $H_{\underline{m}}^{i}(A)=0$ for $i=0,1$, and hence $A \rightarrow$ $H^{0}(\operatorname{Spex}(A), A)$ is an isomorphism. The exact commutative diagram

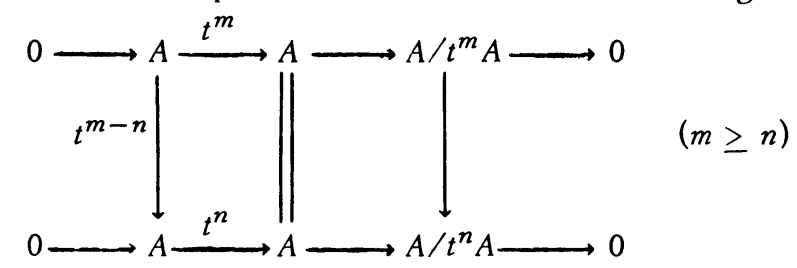

entails the exact commutative diagram

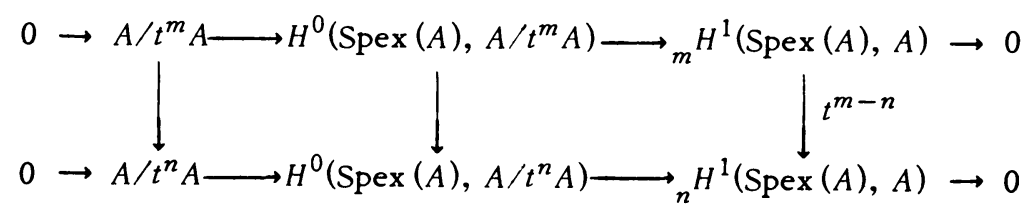

where for any $A$-module $E$ we set ${ }_{n} E=\left\{x \in E \mid t^{n} x=0\right\}=\operatorname{Hom}_{A}\left(A / t^{n} A, E\right)$. Sending to limit we get the exact sequence

$$
0 \rightarrow \underset{v}{\lim } A / t^{v} A \rightarrow \underset{v}{\lim } H^{0}\left(\operatorname{Spex}(A), A / t^{v} A\right) \rightarrow \underset{v}{\lim _{v}} H^{1}(\operatorname{Spex}(A), A)
$$

Now $H_{\underline{m}}^{2}(A)=H^{1}($ Spex $(A), A)$ is coherent by hypothesis and hence there exists an integer ${ }^{\underline{m}} n$ such that ${ }_{n} H^{1}(\operatorname{Spex}(A), A)={ }_{m} H^{1}(\operatorname{Spex}(A), A)$ for all $m \geq n$. Consequently, 


$$
{ }_{m} H^{1}(\operatorname{Spex}(A), A) \stackrel{t^{n}}{\longrightarrow}{ }_{m-n} H^{1}(\operatorname{Spex}(A), A)
$$

is a zero-map for all $m \geq n$, and hence

$$
\varliminf_{v}{ }_{\nu} H^{1}(\operatorname{Spex}(A), A)=0,
$$

and therefore $\lim _{\nu} A / t^{\nu} A \rightarrow \lim _{v} H^{0}\left(\operatorname{Spex}(A), A / t^{\nu} A\right)$ is an isomorphism. In other

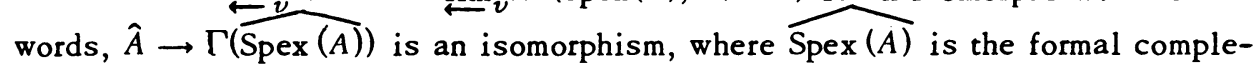
tion of Spex $(A)$ along the closed subscheme Spex $(A / t A)$. Consequently, $\Gamma(\widehat{\operatorname{Spex}(A)})$ and therefore $\Gamma(\operatorname{Spex}(A / t A))$ has no nontrivial idempotent elements and hence Spex $(A / t A)$ is connected.

Proposition 3.3. Let $A_{0}$ be a reduced noetherian local ring with $\operatorname{dim} A_{0} \geq 2$. If there exists a noetherian local ring $A$ which is a bomomorphic image of a regular local ring, and an A-regular element $t$ in the maximal ideal of $A$ sucb that $A / t A$ $\simeq A_{0}$ and $A_{t}$ is regular, then $A_{0}$ is equidimensional and Spex $\left(\hat{A}_{0}\right)$ is connected, where $\hat{A}_{0}$ stands for the completion of $A_{0}$ with respect to the maximal ideal of $A_{0}$.

Proof. Assume that $A$ is normal. Then $t A$ is an unmixed ideal in $A$ and hence $A_{0} \simeq A / t A$ is equidimensional. Let us denote by $\hat{A}$ the completion of $A$ with respect to the maximal ideal $\underline{m}$ of $A$, so that $t$ is $\hat{A}$-regular and $\hat{A} / t \hat{A} \simeq$ $\hat{A}_{0}$. We note that depth $\hat{A}=\operatorname{depth} A \geq 2$ since $A$ is normal and $\operatorname{dim} A \geq 2$. If $H_{\underline{m}}^{2} \hat{A}(\hat{A})$ is coherent, it follows from the above lemma that Spex $\left(\hat{A}_{0}\right)=\operatorname{Spex}(\hat{A} / t \hat{A})$ is connected. We note that, for every $i>0$,

$$
\begin{aligned}
& H_{\underline{m} \hat{A}}^{i}(\hat{A})=\underset{v}{\lim } \operatorname{Ext}_{\hat{A}}^{i}\left(\hat{A} / \underline{m}^{v} \hat{A}, \hat{A}\right) \\
& =\underset{v}{\lim } \operatorname{Ext}_{A}^{i}\left(A / \underline{m}^{v}, A\right) \underset{A}{\otimes} \hat{A}=\underset{\leftarrow}{\lim } \operatorname{Ext}_{A}^{i}\left(A / \underline{m}^{v}, A\right)=H_{\underline{m}}^{i}(A),
\end{aligned}
$$

and thus it suffices to show that $H_{\underline{m}}^{2}(A)$ is coherent. Now let $x$ be a point of $\operatorname{Spec}(A)$ such that $\operatorname{dim}\{\bar{x}\} \leq 1$. Since $\operatorname{dim} A \geq 3$ we must have $\operatorname{dim} A_{x} \geq 2$ and hence depth $A_{x} \geq 2$ since $A$ is normal. It follows from Grothendieck's criteria [6] that $H_{m}^{2}(A)$ is coherent. Thus it suffices to show that $A$ is normal. We use Serre's criteria. Let $x$ be a point in $\operatorname{Spec}(A)$ such that $\operatorname{dim} A_{x}=1$. If $x \notin V(t)$, then $A_{x}$ is regular by hypothesis. If $x \in V(t)$, then $\operatorname{dim}\left(A_{0}\right)_{x}=\operatorname{dim} A_{x} / t A_{x}=0$ so that $\left(A_{0}\right)_{x}$ is a field since $\left(A_{0}\right)_{x}$ is reduced, which entails that $A_{x}$ is regular. Now let $x$ be a point in $\operatorname{Spec}(A)$ such that $\operatorname{dim} A_{x} \geq 2$. If $x \notin V(t)$, then $A_{x}$ is regular and hence depth $A_{x}=\operatorname{dim} A_{x} \geq 2$. Let $x \in V(t)$. Then $t \in \underline{m}_{x}$ and it is an $A_{x}$-regular element such that depth $A_{x} / t A_{x}=\operatorname{depth}\left(A_{0}\right)_{x}>0$ since $A_{0}$ is reduced, and therefore depth $A_{x} \geq 2$. This completes our proof.

Corollary 3.4. Let $X_{0}$ be a reduced algebraic $k$-scheme which is a specialization of a smooth $k$-scheme, where $k$ is an algebraically closed field. Then for 
$x \in X_{0}$ with $\operatorname{dim} \underline{O}_{X_{0}, x} \geq 2$, Spex $\left(\underline{O}_{X_{0}, x}\right)$ is connected. In particular, every point $x \in X_{0}$ such that $\operatorname{dim} \underline{Q}_{X_{0}, x} \geq 2$ and $\operatorname{Spex}\left(\underline{O}_{X_{0}, x}\right)$ is normal is unibranch.

Proof. We may and shall assume that $X_{0}$ is not smooth over $k$. Let $(R, X)$ be a deformation of $X_{0}$ such that $K \otimes_{R} X$ is smooth over $K$ where $K$ is the total ring of fractions of $R$. We may assume that $R=k[[t]]$. Indeed, $D=\{s \in \operatorname{Spec}(R) \mid$ $\kappa(s) \otimes_{R} X$ is smooth over $\left.\kappa(s)\right\}$ is nonempty open in Spec $(R)$ and does not contain the maximal point of Spec $(R)$, and therefore there exists $s \in D$ such that $\operatorname{dim}\{\bar{s}\}=$ 1. Thus if we set $\underline{p}$ to be the prime ideal in $R$ corresponding to $s$, then $\operatorname{dim} R / \underline{p}$ $=1$ and $\kappa(s) \otimes_{R} X$ is smooth over $\kappa(s)$. Let $R^{\prime}$ be the normalization of $R / \underline{p}$. Since $R / \underline{p}$ is complete with $\operatorname{dim} R / \underline{p}=1$ and $k$ is algebraically closed, we conclude that $R^{\prime}=k[[t]]$. Then $\left(R^{\prime}, R^{\prime} \otimes_{R} X\right)$ is a deformation of $X_{0}$ such that $k[[t]] \otimes_{R^{\prime}}\left(R^{\prime} \otimes_{R} X\right)$ is smooth over $k((t))$. We thus assume that $R=k[[t]]$. Now let $x \in X_{0} \subset X$ be a point such that $\operatorname{dim} \underline{O}_{X_{0, x}} \geq 2$. Since $t$ is $\underline{O}_{X, x}$-regular such that $\underline{O}_{X, x} / t \underline{O}_{X, x} \simeq \underline{O}_{X, x}$ and $\left(\underline{O}_{X, x}\right)_{t} \simeq k((t)) \otimes_{k}[[t]] \underline{O}_{X, x}$ is smooth over $k((t))$ and hence is regular, it follows from 3.3 that Spex $\left(\underline{\hat{o}}_{X_{0}, x}\right)$ is connected. As for the second statement, let $\operatorname{dim} \underline{Q}_{X_{0, x}} \geq 2$ and assume that Spex $\left(\underline{O}_{X_{0}, x}\right)$ is normal. It suffices to show that Spex $\left(\underline{O}_{X_{0}, x}\right)$ is connected if and only if $\underline{O}_{X_{0}, x}$ is unibranch. We set $A=\underline{O}_{X_{0, x}}$ and $A^{\prime}$ the normalization of $A$. Since $A^{\prime} / A$ as an $A$ module is annihilated by some power of the maximal ideal of $A$, we get the exact sequence

$$
0 \rightarrow \hat{A} \rightarrow \hat{A}^{\prime} \rightarrow A^{\prime} / A \rightarrow 0
$$

and hence $(\hat{A})_{f} \rightarrow\left(\hat{A}^{\prime}\right)_{f}$ is an isomorphism for every $f$ in the maximal ideal of $A$. Consequently the morphism $\pi: \operatorname{Spec}\left(\hat{A}^{\prime}\right) \rightarrow \operatorname{Spec}(\hat{A})$ induces the isomorphism $\operatorname{Spex}\left(\hat{A}^{\prime}\right) \cong \operatorname{Spex}(\hat{A})$ where Spex $\left(\hat{A}^{\prime}\right)=\operatorname{Spec}(A)-\pi^{-1}\{\underline{\hat{m}}\}, \underline{\hat{m}}=$ the maximal ideal of $\hat{A}$. However, $A^{\prime}$ is semilocal and hence $\hat{A}^{\prime}=A_{1}^{\prime} \times \cdots \times A_{m}^{\prime}$, where $A_{i}^{\prime}$ is a complete local normal domain, and hence $\operatorname{Spex}\left(\hat{A}^{\prime}\right)=\operatorname{Spex}\left(A_{1}^{\prime}\right) \cup \cdots \cup \operatorname{Spex}\left(A_{n}^{\prime}\right)$ (disjoint union). We note that $\operatorname{depth} A_{i}^{\prime} \geq 2$ since it is normal with dimension $\geq 2$, and therefore Spex $\left(A_{i}^{\prime}\right)$ is connected by Hartshorne's lemma. It follows that $\operatorname{Spex}(\hat{A}) \simeq \operatorname{Spex}\left(A_{1}^{\prime}\right) \cup \cdots \cup \operatorname{Spex}\left(A_{n}^{\prime}\right)$ is connected if and only if $n=1$. This completes our proof.

It is now clear how to construct an algebraic variety of dimension $\geq 2$ which can never be a limit of smooth varieties: Let $X$ be a normal variety. Given a 0 dimensional closed subscheme $Y \subset X$, there exists an algebraic variety $X_{\{Y\}}$ together with the birational morphism $\pi: X \rightarrow X_{\{Y\}}$ such that

(i) $\pi(Y)$ consists of one point $z$, and $X-Y \rightarrow{ }^{\pi} X_{\{Y\}}-\{z\}$ is an isomorphism.

(ii) The ideal sheaf $\underline{I}_{Y}$ of $Y$ in $\underline{O}_{X}$ is equal to the conductor ideal sheaf of $\pi$.

If $\operatorname{dim} X \geq 2$ and $Y$ consists of more than one point, then $\operatorname{dim} O_{X\{Y\}, z} \geq 2$ and $z$ is of multiple branches, and consequently $X_{\{Y\}}$ cannot be a specialization of a 
smooth variety. Motivated by this fact, we can now construct, for any integer $n \geq$ 2 , a rigid $n$-dimensional affine variety with an isolated singularity.

Lemma 3.5. Set

$$
\begin{aligned}
A=K & {\left[\left[X_{1}, \cdots, X_{n}\right]\right] \underset{k}{\times} k\left[\left[Y_{1}, \cdots, Y_{n}\right]\right] } \\
& \cong K\left[\left[X_{1}, \cdots, X_{n}, Y_{1}, \cdots, Y_{n}\right]\right] /\left(X_{i} Y_{j} ! 1 \leq i, j \leq n\right) .
\end{aligned}
$$

If $E$ is an A-module of finite type such that depth $E>0$ and $\operatorname{depth} E /\left(x_{i}, y_{j}\right) E>0$ for all $i, j$, then $\operatorname{Ext}_{A}^{1}\left(\hat{\Omega}_{\left.A\right|_{k}}, E\right)=0$, where $\hat{\Omega}_{A \mid k}$ stands for the A-module of continuous k-differentials of $A$. In particular, $\operatorname{Ext}_{A}^{1}\left(\hat{\Omega}_{A \mid k}, A\right)=0$ provided $n \geq 2$.

Proof. We have the exact sequence

$$
0 \rightarrow N \rightarrow A d X_{1} \oplus \cdots \oplus A d X_{n} \oplus A d Y_{1} \oplus \cdots \oplus A d Y_{n} \rightarrow \hat{\Omega}_{\left.A\right|_{k}} \rightarrow 0
$$

where $N$ as $A$-module is generated by the set $\left\{\omega_{i j}\left(=d\left(X_{i} Y_{j}\right)\right)=y_{j} d X_{i}+x_{i} d Y_{j} \mid 1 \leq\right.$ $i, j \leq n\}$. Let $\lambda: N \rightarrow E$ be any $A$-linear map. Since we have the relations

$$
\left\{\begin{array}{l}
x_{k} \omega_{i j}=x_{i} \omega_{k j} \\
y_{k} \omega_{i j}=y_{j} \omega_{i k}
\end{array} \text { for all } i, j, k,\right.
$$

we must have that

$$
\left\{\begin{array}{l}
\left(x_{1}, \cdots, x_{n}\right) \lambda\left(\omega_{i j}\right) \subset x_{i} E \\
\left(y_{1}, \cdots, y_{n}\right) \lambda\left(\omega_{i j}\right) \subset y_{j} E,
\end{array}\right.
$$

i.e. $\underline{m} \lambda\left(\omega_{i j}\right) \subset\left(x_{i}, y_{j}\right) E$ where $\underline{m}=\left(x_{1}, \ldots, x_{n}, y_{1}, \ldots, y_{n}\right)$. Since $\operatorname{depth} E /\left(x_{i}, y_{j}\right) E$ $>0$ by hypothesis, we have $\underline{m} \notin$ Ass $E /\left(x_{i}, y_{j}\right) E$ and therefore $\lambda\left(\omega_{i j}\right) \in\left(x_{i}, y_{j}\right) E$. Hence we set

$$
\lambda\left(\omega_{i j}\right)=y_{j} \alpha_{i j}+x_{i} \beta_{i j}
$$

where $a_{i j}, \beta_{i j}$ are the elements in $E$. Then $(*)$ entails again that

i.e.

$$
\left\{\begin{array}{l}
x_{k}\left(y_{j} \alpha_{i j}+x_{i} \beta_{i j}\right)=x_{i}\left(y_{j} \alpha_{k j}+x_{k} \beta_{k j}\right) \\
y_{k}\left(y_{j} \alpha_{i j}+x_{i} \beta_{i j}\right)=y_{j}\left(y_{k} \alpha_{i k}+x_{i} \beta_{i k}\right),
\end{array}\right.
$$

i.e.

$$
\left\{\begin{array}{l}
x_{k} x_{i} \beta_{i j}=x_{i} x_{k} \beta_{k j} \\
y_{j} y_{k} \alpha_{i j}=y_{j} y_{k} \alpha_{i k},
\end{array}\right.
$$




$$
\left\{\begin{array}{l}
x_{k} x_{i}\left(\beta_{i j}-\beta_{k j}\right)=0 \\
y_{j} y_{k}\left(\alpha_{i j}-\alpha_{i k}\right)=0
\end{array} \text { for all } i, j, k .\right.
$$

If $\left(x_{1}, \ldots, x_{n}\right) E \neq 0$, then depth $\left(x_{1}, \ldots, x_{n}\right) E>0$ since depth $E>0$ by hypothesis, and therefore there exists an $\left(x_{1}, \ldots, x_{n}\right) E$-regular element in $\underline{m}$, say let it be $x_{1}$. Then $x_{1} x_{i}\left(\beta_{i j}-\beta_{i j}\right)=0$ for all $i$ entails that $x_{i}\left(\beta_{i j}-\beta_{i j}\right)=0$ for all $i$, i.e. $x_{i} \beta_{i j}=x_{i} \beta_{i j}$ and therefore we may assume that $\beta_{i j}=0$ for all $i, j$. Therefore, in either situation, we may assume in the expression (**) that $\beta_{i j}$ depends only on $j$. Likewise we may assume that $\alpha_{i j}$ depends only on $i$. We thus conclude that there exist $\alpha_{1}, \cdots, \alpha_{n}, \beta_{1}, \cdots, \beta_{n}$ in $E$ such that $\lambda\left(\omega_{i j}\right)=y_{j} \alpha_{i}+x_{i} \beta_{j}$. This simply means that

$$
\operatorname{Ext}_{A}^{1}\left(\hat{\Omega}_{A \mid k}, E\right)=\operatorname{Coker}\left(\operatorname{Hom}_{A}(F, A) \rightarrow \operatorname{Hom}_{A}(N, A)\right)=0
$$

where $F=A d X_{1} \oplus \ldots \oplus A d X_{n} \oplus A d Y_{1} \oplus \ldots \oplus A d Y_{n}$. The second statement is immediate since depth $A>0$ provided $n \geq 1$ and depth $A /\left(x_{i}, y_{j}\right)>0$ provided $n \geq 2$.

Corollary 3.6. Let $X=A_{k}^{n}$ (= the affine $n$-space over $k$ ) where $n \geq 2$, and let $Y=\left\{z_{1}, z_{2}\right\}$ be a reduced closed subscheme of $X$ consisting of two distinct $k$ rational points $z_{1}, z_{2}$. Then $X_{\{Y\}}=\operatorname{Spec}\left(k+\underline{m}_{z_{1}} \cap \underline{m}_{z_{2}}\right)$ is a rigid irreducible $n$ dimensional affine variety with the isolated nonsmooth point.

Proof. The morphism $\pi: X \rightarrow X_{\{Y\}}$ induces by definition an isomorphism $X-$ $Y \rightarrow X_{\{Y\}}-\{z\}$ where $z=\pi(Y)$, and therefore $X_{\{Y\}}$ is smooth over $k$ except at the point $z$. Set $X_{\{Y\}}=\operatorname{Spec}(A)$. Since $A$ is smooth over $k$ except at the point $z, \Omega_{A \mid k}$ is locally-free everywhere except at the point $z$ and therefore $\operatorname{Ext}_{A}^{1}\left(\Omega_{A \mid k}, A\right)$ is an $A$-module of finite type annihilated by some power of $\underline{m}_{z}$. Consequently the canonical maps

$$
\operatorname{Ext}_{A}^{1}\left(\Omega_{A \mid k^{\prime}}, A\right) \rightarrow \operatorname{Ext}_{A_{z}}^{1}\left(\Omega_{A_{z}} \mid k^{\prime}, A_{z}\right) \rightarrow \operatorname{Ext}_{\hat{A}_{z}}^{1}\left({\widehat{\Omega_{\hat{A}_{z} \mid k}}}_{\hat{A}_{z}}\right)
$$

are all isomorphisms, where $\widehat{\Omega_{\hat{A}} \mid k}$ stands for the module of continuous $k$-differentials of $\hat{A}_{z}$. However, we have by definition the exact sequence

$$
0 \rightarrow A_{z} \rightarrow \underline{O}_{X, z} \stackrel{z_{1}-z_{2}}{\longrightarrow} k \rightarrow 0
$$

where $\underline{O}_{X, z}=\underline{\lim }_{U} \supset_{Y} \Gamma^{\prime}\left(U, \underline{O}_{X}\right)$ is the semilocal ring with two distinct maximal ideals corresponding to the points $z_{1}, z_{2}$. Passing to the completion we get the exact sequence

$$
0 \rightarrow \hat{A}_{z} \rightarrow \underline{\hat{O}}_{X, z_{1}} \times \underline{\hat{O}}_{X, z_{2}} \stackrel{z_{1}-z_{2}}{\longrightarrow} k \rightarrow 0
$$


and therefore $\hat{A}_{z} \simeq \hat{\underline{O}}_{X, z_{1}} \times_{k} \hat{\underline{O}}_{X, z_{2}} \simeq k\left[\left[X_{1}, \ldots, X_{n}\right]\right] \times_{k} k\left[\left[Y_{1}, \ldots, Y_{n}\right]\right]$. It follows from 3.5 that $\operatorname{Ext} \hat{A}_{A \mid k}^{1}\left(\Omega_{\left.\right|_{k}}, A\right) \simeq \operatorname{Ext} \hat{A}_{z}^{1}\left({\widehat{\Omega_{\hat{A}}}}_{z}, \hat{A}_{z}\right)=0$ provided $n \geq 2$, which completes our proof.

4. Comments. In this paper we have studied the deformations of projective curves which is locally a complete-intersection. Admittedly it is the simplest situation since the local obstructions for the prolongations of deformation do vanish by virtue of a property of complete-intersection [1]. However, we have examined the deformation problems of several curves which is not a complete-intersection including the ordinary multiple point with mutually transversal tangents. Based on 2.7 and a few empirical results, we make the following conjectures.

Let $k$ be a fixed perfect field (or an algebraically closed field if necessary) of any characteristic, and let $X_{0}$ be an irreducible reduced complete curve over $k$, and let $(R, X)$ be a Schlessinger deformation of $X_{0}$. The complete local $k$-algebra $R$ is in general not a formal power-series ring even when $X_{0}$ has ordinary multiple points (with mutually transversal tangents) only (see [9]).

$$
s_{X_{0}}=3 g-3+\operatorname{dim}_{k} H^{0}\left(X_{0}, \underline{\Omega}_{X_{0}}^{*}\right) \text { where } g=\operatorname{dim}_{k} H^{1}\left(X_{0}, \underline{O}_{X_{0}}\right) .
$$

One notes that the above formula coincides with the original conjecture of $P$. Deligne [3] in the case when the base field $k$ is of characteristic zero. In view of 2.6, the conjecture (I) is entirely of local nature. Indeed, we set, for each point $x \in X_{0}$,

$$
s_{x}=s_{\operatorname{Spec}}\left(\underline{O}_{X_{0}, x}\right) \cdot
$$

Then $s_{x}=0$ at every smooth point $x$, and 2.6 entails that $s_{X_{0}}=\operatorname{dim}_{k} H^{1}\left(X_{0}, \underline{\Omega}_{X_{0}}^{*}\right)$ $+\sum_{x} s_{x}$. Therefore,

$$
\begin{aligned}
s_{X_{0}} & =3 g-3+\operatorname{dim}_{k} H^{0}\left(X_{0}, \underline{\Omega}_{X_{0}}^{*}\right) \\
& \Leftrightarrow 3 g-3+\operatorname{dim}_{k} H^{0}\left(X, \underline{\Omega}_{X_{0}}^{*}\right)=\operatorname{dim}_{k} H^{1}\left(X_{0}, \underline{\Omega}_{X_{0}}^{*}\right)+\sum_{x} s_{x} \\
& \Leftrightarrow X\left(\underline{\Omega}_{X_{0}}^{*}\right)=3-3 g+\sum_{x \in X_{0}} s_{x} .
\end{aligned}
$$

If we denote by $\pi: X_{0}^{\prime} \rightarrow X_{0}$ the normalization of $X_{0}$, it follows from the RiemannRoch theorem that $\chi\left(\underline{\Omega}_{X_{0}^{\prime}}^{*}\right)=3-3\left(g-\delta_{X_{0}}\right)$ where

$$
\delta_{X_{0}}=\operatorname{dim}_{k} H^{0}\left(X_{0}, \pi_{*} \underline{O}_{X_{0}^{\prime}} / \underline{O}_{X_{0}}\right), \quad \underline{\Omega}_{X_{0}^{\prime}}^{*}=\underline{\operatorname{Hom}}_{\underline{O}_{X_{0}^{\prime}}}\left(\underline{\Omega}_{X_{0}^{\prime}}, \underline{O}_{X_{0}^{\prime}}\right)
$$

Now let

$$
\mathcal{C}_{X_{0}}=\underline{\operatorname{Hom}}_{\underline{O}_{X_{0}}}\left(\pi_{*} \underline{O}_{X_{0}^{\prime}}, \underline{O}_{X_{0}}\right)
$$

be the conductor ideal sheaf of $X_{0}$. We then have canonical inclusion maps 
$(*)$

$$
\left\{\begin{array}{l}
0 \rightarrow \underline{\operatorname{Hom}}_{\underline{O}_{X}}\left(\pi_{*} \underline{\Omega}_{X_{0}^{\prime}}, \underline{\mathcal{C}}_{X_{0}}\right) \rightarrow \pi_{*}\left(\underline{\Omega}_{X_{0}^{\prime}}^{*}\right) \\
0 \rightarrow \underline{\operatorname{Hom}}_{\underline{O}_{X_{0}}}\left(\pi_{*} \underline{\Omega}_{X_{0}^{\prime}}, \underline{\mathcal{C}}_{X_{0}}\right) \rightarrow \underline{\Omega}_{X_{0}}^{*}
\end{array}\right.
$$

For each point $x \in X_{0}$, we set

$$
\begin{aligned}
& d_{x}=\operatorname{dim}_{k}\left[\operatorname{Coker}\left(\underline{\operatorname{Hom}}_{\underline{O}_{X}}\left(\pi_{*} \underline{\Omega}_{X_{0}^{\prime}}, \underline{\mathcal{C}}_{X_{0}}\right) \rightarrow \underline{\Omega}_{X_{0}}^{*}\right)\right]_{x}, \\
& c_{x}=\operatorname{dim}_{k}\left(\pi_{*} \underline{O}_{X_{0}^{\prime}} / \underline{\mathcal{C}}_{X_{0}}\right)_{x} .
\end{aligned}
$$

We note that, for each point $x \in X_{0}$,

$$
\operatorname{dim}_{k}\left[\operatorname{Coker}\left(\underline{\operatorname{Hom}}_{X_{X}}\left(\pi_{*} \underline{\Omega}_{X_{0}^{\prime}}, \underline{\mathcal{C}}_{X_{0}}\right) \rightarrow \pi_{*}\left(\underline{\Omega}_{X_{0}^{\prime}}^{*}\right)\right)\right]_{x}=\operatorname{dim}_{k}\left(\pi_{*} \underline{O}_{X_{0}^{\prime}} / \underline{\mathcal{C}}_{X_{0}}\right)_{x}=c_{x},
$$

and that $d_{x}=0=c_{x}$ at every smooth point $x$. Now the inclusion maps $(*)$ entails that $\chi\left(\underline{\Omega}_{X}^{*}\right)=\chi\left(\underline{\Omega}_{X_{0}}^{*}\right)+\sum_{x} c_{x}-\sum_{x} d_{x}$, and therefore we get $3-3\left(g-\delta_{X_{0}}\right)=$ $\chi\left(\underline{\Omega}_{X_{0}}^{*}\right)+\sum_{x}\left(c_{x}-d_{x}\right)$, i.e. $\chi\left(\underline{\Omega}_{X_{0}}^{*}\right)=3-3 g+3 \delta_{X_{0}}+d_{X_{0}}-c_{X_{0}}$ where $d_{X_{0}}=$ $\sum_{x} d_{x}, c_{X_{0}}=\sum_{x} c_{x}=\operatorname{deg} \underline{\mathcal{C}}_{X_{0}}$. Therefore, $\chi\left(\underline{\Omega}_{X_{0}}^{*}\right)=3-3 g+\sum_{x} s_{x} \Leftrightarrow \sum_{x} s_{x}=$ $3 \delta_{X_{0}}+d_{X_{0}}-c_{X_{0}}$, i.e. the conjecture (I) holds if and only if $\sum_{x} s_{x}=$

$\sum_{x}\left(3 \delta_{x}+d_{x}-c_{x}\right)$. Taking complete curves with a single singular point, we conclude that the conjecture (I) holds if and only if $s_{x}=3 \delta_{x}+d_{x}-c_{x}$ for every (singular) point $x$. Therefore one can give the local reformulation of the above conjecture as follows.

(I) ' Let $A_{0}$ be any 1-dimensional geometric local domain over $k$. Set $A_{0}^{\prime}$ to be the normalization of $A_{0}$, and $C$ the conductor ideal of $A$. Then $s_{\operatorname{Spec}\left(A_{0}\right)}=$ $3 \delta+d-c$ where

$$
\begin{aligned}
& \delta=\operatorname{dim}_{k} A_{0}^{\prime} / A_{0}, \quad c=\operatorname{dim} A_{0}^{\prime} / \mathcal{C}, \\
& d=\operatorname{dim}_{k} \operatorname{Coker}\left(\operatorname{Der}_{k}\left(A_{0}^{\prime}, \mathcal{C}\right) \rightarrow \operatorname{Der}_{k}\left(A_{0}, A_{0}\right)\right) .
\end{aligned}
$$

Remark. It follows from a well-known inequality $\delta \leq c \leq 2 \delta$ that $\delta+d \leq 3 \delta+$ $d-c \leq 2 \delta+d$, and in particular $3 \delta+d-c=0$ if and only if $\delta=0$, i.e. $A_{0}$ is smooth over $k$. We also note that if $A_{0}$ is Gorenstein then $3 \delta+d-c=\delta+$ $\operatorname{dim} \Omega_{A_{0}^{\prime} / i}\left(\Omega_{A_{0}}\right)$.

(II) Let $X_{0}$ be Gorenstein. Then for each integer $0 \leq p \leq \operatorname{dim} \omega_{X_{0} \mid k}^{t}$, $I_{R}\left(\bigwedge^{p} \omega_{X \mid R}^{t}\right)$ is an unmixed ideal of codimension $p$, where the exterior: power is taken over $R$.

Needless to say, (II) is equally a local problem. (II) implies in particular that for every integer $0 \leq m \leq \operatorname{dim} \omega_{X}^{t} \mid k,\left\{z \in \operatorname{Spec}(R)\left|\operatorname{dim} \omega_{X(z)}^{t}\right| \kappa(z)=m\right\}$ is a nonempty locally closed subset of $\operatorname{Spec}(R)$. In case when (II) is correct, one can make the following conjecture: We set, for éach complete curve $Y, l_{Y}=s_{Y}-\operatorname{dim} H^{1}\left(Y, \Omega_{Y}^{*}\right)$. One may note that if $Y$ is Gorenstein and the conjecture (I) is valid, then $l_{Y}=\operatorname{dim} \omega_{Y}^{t}$. 
(III) $l: \operatorname{Spec}(R) \rightarrow N$ given by $z \rightarrow l_{X(z)}$ is an upper semicontinuous function, and for each integer $0 \leq p \leq l_{X_{0}}, \Sigma(p)=\left\{z \in \operatorname{Spec}(R) \mid l_{X(z)} \geq p\right\}$ is an equidimensional closed subset of codimension $p$, where $X(z)=\kappa(z) \otimes_{R} X$.

One may note that (III) certainly would imply that every complete curve is a specialization of a nonsingular curve. The above conjecture (III) has been verified for curves with ordinary multiple points with mutually transversal tangents (see [9]).

(IV) Assume that $X_{0}$ is affine. Given a point $z \in \operatorname{Spec}(R),\left(R_{z}, R_{z} \otimes_{R} X\right)$ is a versal deformation of the curve $X(z)=\kappa(z) \otimes_{R} X$.

This conjecture is also correct for curves with ordinary multiple points with mutually transversal tangents. We remark that $\left(R_{z}, R_{z} \otimes_{R} X\right)$ is not in general a Schlessinger deformation of $X(z)$ and that (IV) is false for complete curves. The conjecture (IV) will have the following significant consequence:

(IV)' If $X_{1} \rightarrow X_{0}$ is a nontrivial deformation of complete curves, then $l_{X_{1}}<$ $l_{X_{0}}$

\section{BIBLIOGRAPHY}

1. M. André, Méthode simpliciale en algèbre homologique et algèbre commutative, Lecture Notes in Math., vol. 32, Springer-Verlag, New York, 1967. MR 35 \#5493.

2. D. A. Buchsbaum and D. S. Rim, A generalized Koszul complex. II: Depth and multiplicity, Trans. Amer. Math. Soc. 111 (1964), 197-224. MR 28 \#3076.

3. P. Deligne, Intersections sur les surfaces régulières, Séminaire de Géométrie Algébrique, vol. 7, Institut des Hautes Études Scientifique, Paris, 1969.

4. H. Grauert and H. Kerner, Deformationen von Singularitäten komplexer Räume, Math. Ann. 153 (1964), 236-260. MR 30 \#592.

5. A. Grothendieck, Géométrie formelle et géométrie algébrique, Séminaire Bourbaki, 1958/59, Fasc. 3, exposé 182, Secrétariat mathématique, Paris, 1959.

6. - Cohomologie locale des faisceaux cohérents et théorèmes de Lefschetz locaux et globaux, (SGA 2) Séminaire de Géométrie Algébrique du Bois-Marie, 1962, Advanced Studies in Pure Math., vol. 2, North-Holland, 1968.

7. R. Hartshorne, Residues and duality, Lecture Notes in Math., no. 20, Springer-Verlag, New York, 1966. MR 36 \#5145.

8. D. S. Rim, On formal moduli of deformations, Séminaire de Géométrie Algébrique, vol. 7 (to appear).

9. - Deformations of ordinary multiple points (to appear).

10. M. Schlessinger, Functors of Artin rings, Trans. Amer. Math. Soc. 130 (1968), 208222. MR $36 \# 184$.

11. Anonymous, Correspondence, Amer. J. Math. 79 (1957), 951-952.

DEPARTMENT OF MATHEMATICS, UNIVERSity OF PENNSYLVANIA, PHILADELPHIA, PENNSYLVANIA 19104 